\title{
CYCLOIDAL GAIT WITH DOUBLE SUPPORT PHASE FOR THE NAO HUMANOID ROBOT
}

\author{
Jesus E. Fierro P. \\ Researcher \\ Division of Postgraduate Studies and Research ${ }^{1}$ \\ jexfp1@gmail.com \\ J. Alfonso Pamanes G. \\ Full Professor \\ Division of Postgraduate Studies and Research ${ }^{l}$ \\ japamanesg@correo.itlalaguna.edu.mx \\ Victor De-Leon-Gomez \\ Full Professor \\ Faculty of Administration \\ Universidad Autonoma de Coahuila \\ 247 Revolucion blvd., Torreon, Coah. Mexico, 27000 \\ vsdeleon@hotmail.com
}

${ }^{1}$ Tecnologico Nacional de Mexico / Instituto Tecnologico de la Laguna

Revolucion y Calz. Cuauhtemoc blvd., Torreon, Coah. Mexico, 27000

\begin{abstract}
The commercial Nao humanoid robot has 11 DOF in legs. Even if these legs include 12 revolute joints, only 11 actuators are employed to control the walking of the robot. Under such conditions, the mobility of the pelvis and that of the oscillating foot are mutually constrained at each step. Besides, the original gait provided by the manufacturer company of the Nao employs only single support phases during the walking. Because of both issues, the reduced mobility in legs and the use of only single support phases, the stability of the walking is affected. To contribute to improving such stability, in this paper an approach is proposed that incorporates a double support phase and a gait based on cycloidal time functions for motions of the pelvis and those of the oscillating foot. To assess the stability of the walking an index is applied, which is based on the notion of zero-moment point (ZMP) of the static foot at each step. Results of experimental tests show that the proposed gait enhances the stability of the robot during the walking.
\end{abstract}

Keywords: Nao humanoid robot, gait, stability, cycloidal walking pattern, single support phase, double support phase.

DOI: $10.21303 / 2461-4262.2019 .001057$

\section{Introduction}

Most of the humanoid robots studied in the scientific literature have kinematic chains with 6 degrees of freedom (DOF) per leg so that the poses (i. e. positions and orientations) of both the torso and the oscillating foot can be fully controlled in the 3D space during the walking [1-7]. However, the popular Nao humanoid robot has only 11 DOF in the legs [8]. Even if this robot has 12 revolute joints in the legs, it uses only 11 motors to control the joint motions. Indeed, the rotations of the sixth and seventh joints are controlled by using only one actuator. Under such conditions, the 3D motion of the torso and the oscillating foot cannot be independently specified. Thus, the mobility of these bodies is mutually constrained. On the other hand, the original gait provided by the manufacturer company of the Nao uses only a single support phase (SSP) during a step of the walking. Consequently, getting stable gaits in the robot becomes a complex issue.

In contrast with a walking based only on SSP that one centered on both, single and double support phases, improve the stability of walking. SSP implicates only one static foot whereas the double support phase (DSP) involves two static feet during a step. The double support period allows avoiding jumps of accelerations of bodies in such a way that the ZMP smoothly transfers from the former support to the new support [9]. On the other hand, in the specific case of the Nao an advantage could be taken of the DSP because, during the period employed for that phase, the conditions 
for an elemental walking of the robot [10] could be accomplished. That is why in this paper a strategy based on the inclusion of a DSP is considered to try to improve the stability of the Nao's walking. Additionally, a gait based on cycloidal functions for translational and rotational displacements of the torso and the free foot will be implemented. As a result, a more stable and efficient walking of the robot is gotten.

\section{Material and Methods}

\section{1. The Nao humanoid robot}

The Nao robot has 25 DOF distributed as follows: five in each arm, one in each hand, two for the head, and eleven for the legs. The first public version of this robot was presented in 2008 [8]. It is $0.57 \mathrm{~m}$ tall and about $4.5 \mathrm{~kg}$ of weight. Fig. 1 shows the kinematic scheme of the robot's legs.

Each leg has six revolute joints however, the rotations in the two joints attached to the pelvis are produced by only one actuator. Indeed, these motions are constrained in such a way that reciprocal turnings must be always accomplished.

Some strategies for gait planning have been proposed in the literature for the Nao robot e.g. [11], however, the technique applied by Aldebaran to the humanoid is based on the inverse pendulum method [12] in such a way that rectilinear paths are followed by the robot. A gait obtained for the Nao by applying this technique will be termed Aldebaran gait in this paper. It must be observed that such a gait is not explicitly known in terms of motions of the pelvis and the oscillating foot but as a result of the inverse pendulum control technique that determines the control signals for the joint variables.

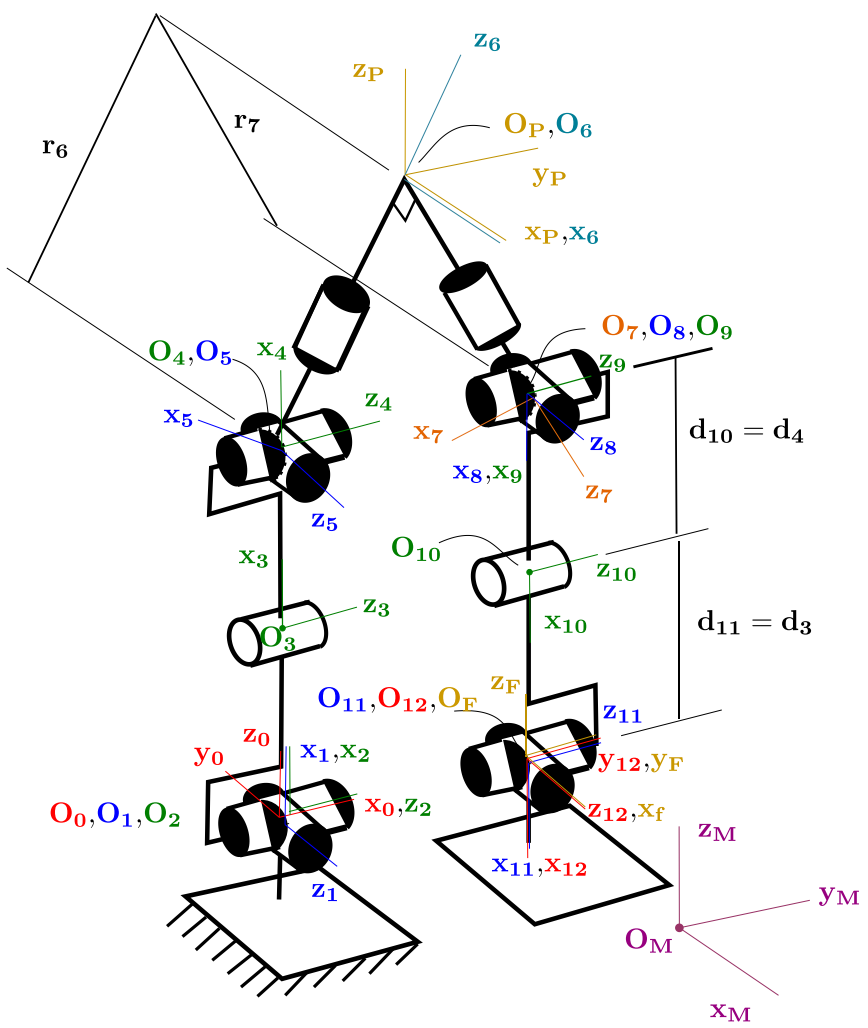

Fig. 1. Kinematic scheme of legs of the Nao robot (static right foot: srf)

The Aldebaran Robotics Company developed the Nao robot to achieve a low-cost platform supplemented by its communication library. The programming of the Nao is based on the application of Naoqi, a framework that can be employed in programming languages like $\mathrm{C}++$, Python, or Java. The main software development tool is Choregraph [13] a blocks programming language. For physical simulation, the software of Nao employs Webots for Nao, a special version of We- 
bots [14] compatible with Choregraph. The Nao robot includes systems to measure positions and current on each joint.

\section{2. Kinematics of the robot}

The gait of a humanoid robot is specified by the desired translational and rotational motions of the pelvis and the oscillating foot during the walking. Clearly, a gait is associated with the robot's walking pattern that is defined by the reference control signals of its joint variables. The desired poses of each body should be described by six time-functions: three for position and three for orientation. In a previous work [15] an interesting family of cycloidal functions was proposed to specify a 3D gait of a humanoid robot. Such functions are applied in this work to the Nao to describe the Cartesian coordinates for position and the Bryant angles for orientation of the orthonormal frames $\Sigma_{P}\left(O_{P} x_{p} y_{p} z_{p}\right)$, attached to the pelvis, and $\Sigma_{f}\left(O_{f} x_{f} y_{f} z_{f}\right)$, attached to the oscillating foot. Both poses with respect to a frame $\Sigma_{M}\left(O_{M} x_{M} y_{M} z_{M}\right)$ attached to the ground. These frames are shown in Fig. 1. The Bryant angles $\lambda, \mu, v$ are those of three successive rotations applied to the reference frame $\Sigma_{M}$ about the axes $x, y, z$, respectively, in order to match the orientation of the frame to be described $\left(\Sigma_{P}\right.$ or $\left.\Sigma_{f}\right)$. The physical meaning of the parameters that define the linear motion of the robot is shown in Fig. 2.

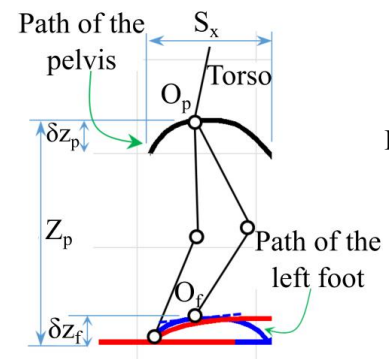

$a$

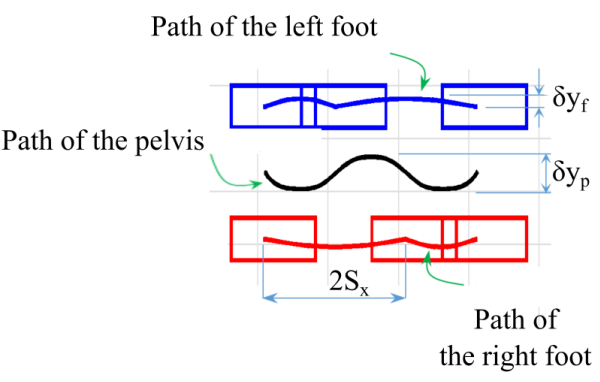

$b$

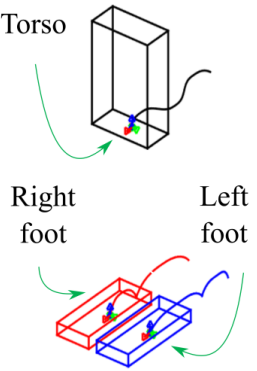

c

Fig. 2. Main parameters of linear motion of a cycloidal gait for a humanoid [13]: $a$ - Side view, $b$ - Top view, $c$ - Isometric view

To model the kinematic chain of the Nao robot, this one is described by employing the modified version of the Denavit-Hartenberg method given in [16]. At each step of the robot, the successive links are numbered from 0 for the static foot to 12 for the oscillating foot. Orthonormal reference frames are attached to the links and are also numbered, as shown in Fig. 1 for the static right foot $(s r f)$. Let's note that the numbering of links and frames is inverted for the static left foot (slf) and consequently a kinematic model is obtained for srf and another one for slf. Then, the geometric parameters that define the pose of the frame $\Sigma_{i}\left(O_{i} x_{i} y_{i} z_{i}\right)$ with respect to the frame $\Sigma_{i-1}$ $\left(O_{i-1} x_{i-1} y_{i-1} z_{i-1}\right)$ are identified $(i=1,2, \ldots, 12)$. The joint variable $\theta_{i}$ is the angle from $x_{i-1}$ to $x_{i}$ about $z_{i}$. The supplementary frame $\Sigma_{P}\left(O_{P} x_{P} y_{P} z_{P}\right)$ attached to the pelvis is rotated by a constant angle $\theta_{P}$ about the $x_{6}$ axis of the $\Sigma_{6}$ frame.

The kinematic models are presented in [17]. They are employed in simulations of the kinematics of the robot in this paper. The numerical values in millimetres for the distances shown in Fig. 1 are: $d_{3}=d_{11}=103 ; d_{4}=d_{10}=100$ and $r_{6}=r_{7}=71.2$. Besides, $\theta_{P}=45^{\circ}$ for srf and $\theta_{P}=-45^{\circ}$ for slf. The full list of the geometric parameters can be observed in [15]. Because of the constraint in joints attached to the pelvis, the relationship (1) holds during the walking between the joint variables $\theta_{6}$ and $\theta_{7}$ associated with the $6^{\text {th }}$ and the $7^{\text {th }}$ joints respectively:

$$
\theta_{6}=\theta_{7}
$$

An essential issue to take into account in specifying the parameters of a cycloidal gait of the Nao robot is associated with the constraint (1). As previously indicated, the twelve operational coordinates that describe the poses of the pelvis and the oscillating foot cannot be arbitrarily specified 
because the legs don't have enough DOF to control all of them. So, only a part of such coordinates and the corresponding parameters should be specified.

\section{3. Stability of the Nao during the walking}

The behaviour of the zero-moment point (ZMP) [18] on the static feet is applied in this paper to measure the stability of the humanoid robot while walking. During a period of simple support, the closer the set of the zero-moment points to the centroid $C_{0}$ of the footprint is, the better is the stability of the robot. To measure the distance of such a set, the coordinates corresponding to a sample of $n_{m p}$ zero-moment points $P_{j}$ are considered $\left(j=1, \ldots, n_{m p}\right)$, which correspond to the $k$-th step $\left(k=1, \ldots, n_{w s}\right)$ in SSP during a walking of $n_{w s}$ steps. These coordinates are obtained in a physical simulation of the robot by using Webots for Nao. Then, the $\delta_{j}$ distances are computed between each $P_{j}$ point of the sample and the centroid $C_{0}$ of the sole of the support foot. The zero moment points of the set and $C_{0}$ are schematized in the sole of the humanoid in Fig. 3, and a distance $\delta_{j}$ is also represented.

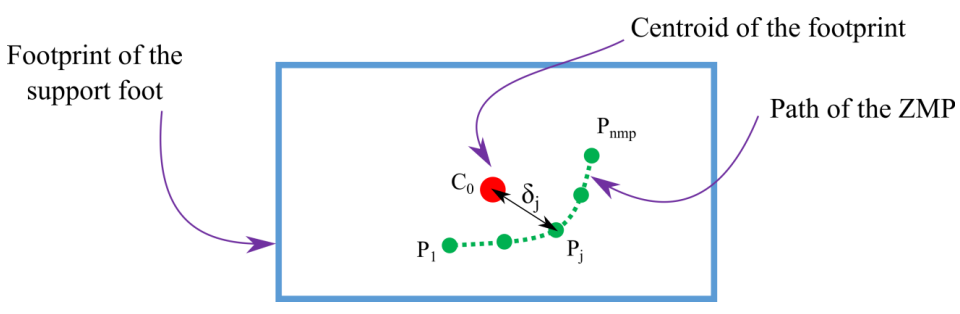

Fig. 3. Sample of zero moment points $P_{j}$ for the $k$-th step of a walking

The mean value $\bar{\delta}$ and the standard deviation $\delta_{\sigma}$ of the set of distances $\delta_{j}$ are computed and replaced in the following function:

$$
\delta_{k}^{*}=\bar{\delta}+\delta_{\sigma},
$$

where

$$
\begin{gathered}
\bar{\delta}=\frac{1}{n_{m p}} \sum_{i=1}^{n_{m p}} \delta_{i}, \\
\delta_{\sigma}=\sqrt{\frac{1}{n_{m p}} \sum_{i=1}^{n_{m p}}\left(\bar{\delta}-\delta_{i}\right)^{2}} .
\end{gathered}
$$

The function $\delta_{k}^{*}$ defined by (2) characterizes a typical distance of the sample of zero moment points from the centroid of the footprint at the $k$-th step of the walking. Thus, such a function can be employed as a measure of the stability of the robot during the step. On the other hand, by taking into account that a walking should be completed by $n_{w s}$ steps, and the values of $\delta_{k}^{*}$ are known at each step, then the numerical value of a global measure $\delta_{w}^{* s}$ can also be computed to assess the stability of the robot associated with a foot in the whole walking by applying the following function:

$$
\delta_{w}^{*}=\overline{\delta^{*}}+\delta_{\sigma}^{*}
$$

where $\overline{\delta^{*}}$ is the mean value and $\delta_{\sigma}^{*}$ is the standard deviation of the set of $\delta_{k}^{*}\left(k=1, \ldots, n_{w s}\right)$ corresponding to the steps of the foot in the whole walking.

\section{Results: analysis of gaits}

To carry out the approach proposed in this paper, three gaits employed for a specific walking are analysed. The first one is the original Aldebaran gait. The others are cycloidal gaits specified 
with different sets of numerical values of parameters for motions of the pelvis and the oscillating foot. In the considered walking the Nao must go through $1000 \mathrm{~mm}$ on a rectilinear path with a speed of $54.945 \mathrm{~mm} / \mathrm{sec}$. This speed was established by taking into account the sampling rate available in Choregraphe to acquire data from a walking.

The gait of Case 1, called Simulated Aldebaran Gait, is focussed on the identification of parameters of cycloidal functions that approximate the motions of the pelvis and the oscillating foot of the original Aldebaran gait, which apply only SSP. In the gait of Case 2, named DS Cycloidal Gait (DS means double support), the found parameters of Case 1 are modified in such a way that both SSP and DSP are employed preserving the stability of the walking. Finally, in the gait of Case 3, called EDS Cycloidal Gait (EDS means enhanced double support), the parameters of Case 2 are still modified in such a way that the stability of the robot during the walking be improved compared with the precedent cases.

\section{1. Simulated Aldebaran Gait}

In the robot programming of motion for Case 1, control signals for the 12 joints variables are generated by using Choregraphe based on the inverse pendulum method and taking into account the constraint (1). Then, during a simulation of the walking in Choregraphe, such control signals are acquired by employing a sampling rate of about $14 \mathrm{~Hz}$. This sample of control signals is then supplied to the software developed in Matlab for motion simulation of the robot. From the results, the parameters of cycloidal functions are identified to simulate the Aldebaran gait in Matlab as a reference gait. The cycloidal functions are defined for three stages of the walking: starting, cruising and stopping. To start the walking, a half-cycloidal starting function is applied to the pelvis during the first step until reaching the cruising speed. Then such a body has to preserve a constant speed during the cruising stage. Finally, in the course of the last step, a half-cycloidal stopping function is specified to the pelvis until getting the resting at the last pose.

The points of the kinematic chain whose linear motions must be specified are $O_{P}$ for the pelvis, and $O_{f}$ for the oscillating foot (Fig. 1). The periods obtained for the three stages are $2 \mathrm{sec}$ for starting and stopping and 14.2 for cruising.

In the three cases study the specified Cartesian coordinates with respect to the frame $\Sigma_{M}$ of $O_{P}$ and $O_{f}$ at the starting point are: $x_{O P}=0, y_{O P}=-50, \quad z_{O P}=234.7, x_{O f}=0(r f$ and $l f), y_{O f}=0(l f)$, $y_{O f}=-100(r f), z_{O f}=0(r f$ and $l f), S_{x}=30$. All units are in millimetres (lf means left foot and $r f$ means right foot). The orientations of the torso (attached to the pelvis) and that of the free foot in the starting posture of the robot are such that the frames $\Sigma_{P}$ and $\Sigma_{f}$ are parallel to the frame $\Sigma_{M}$.

\section{2. Cycloidal gaits}

Taking into account the behaviour observed in Case 1, a process is considered to try to improve the stability of the Nao's walking in cases 2 and 3. This process is illustrated in Fig. 4. It is based on the specification of cycloidal gaits in such a way that the desired rectilinear path of the walking is accomplished. Let's note in Fig. 4 that some activities of the process are achieved by employing different software platforms (Matlab and Webots for Nao), and finally the experimental tests are carried out by using the Nao robot. Improvements in the stability of the Nao's gait could be obtained by testing different sets of numerical values of parameters for the cycloidal gait and including a DSP at each step. For the studies reported here a period $T_{d s}=0.11 \mathrm{sec}$ was employed which corresponds to about $16 \%$ of the period of one step. As a reference to take this value, some amounts reported in the literature were taken into account. Indeed, periods of $20 \%$ and $15 \%$ of one step have been reported for human walking [19] and a humanoid robot [20], respectively.

An essential issue to consider in defining parameters of a cycloidal gait is associated with the constraint (1). Indeed, such a constraint complicates the specification of the ideal poses of the pelvis and the oscillating foot.

The following coordinates are considered as those of the highest priority: those of positions of $O_{P}$ and $O_{f}$, those of orientation of the pelvis, those of the yaw and roll of the oscillating foot. Consequently, the pitch of this foot is not controlled and a perturbation could occur when this foot arrives at the ground or takes off the ground. Certainly, such a perturbation already arise in the 
original Aldebaran gait. It is the main source of instability. It must be observed that during the proposed double support phase the aforesaid perturbation could decrease because the ideal orientations of feet would be compatible with that of the pelvis during the period $T_{d s}$.

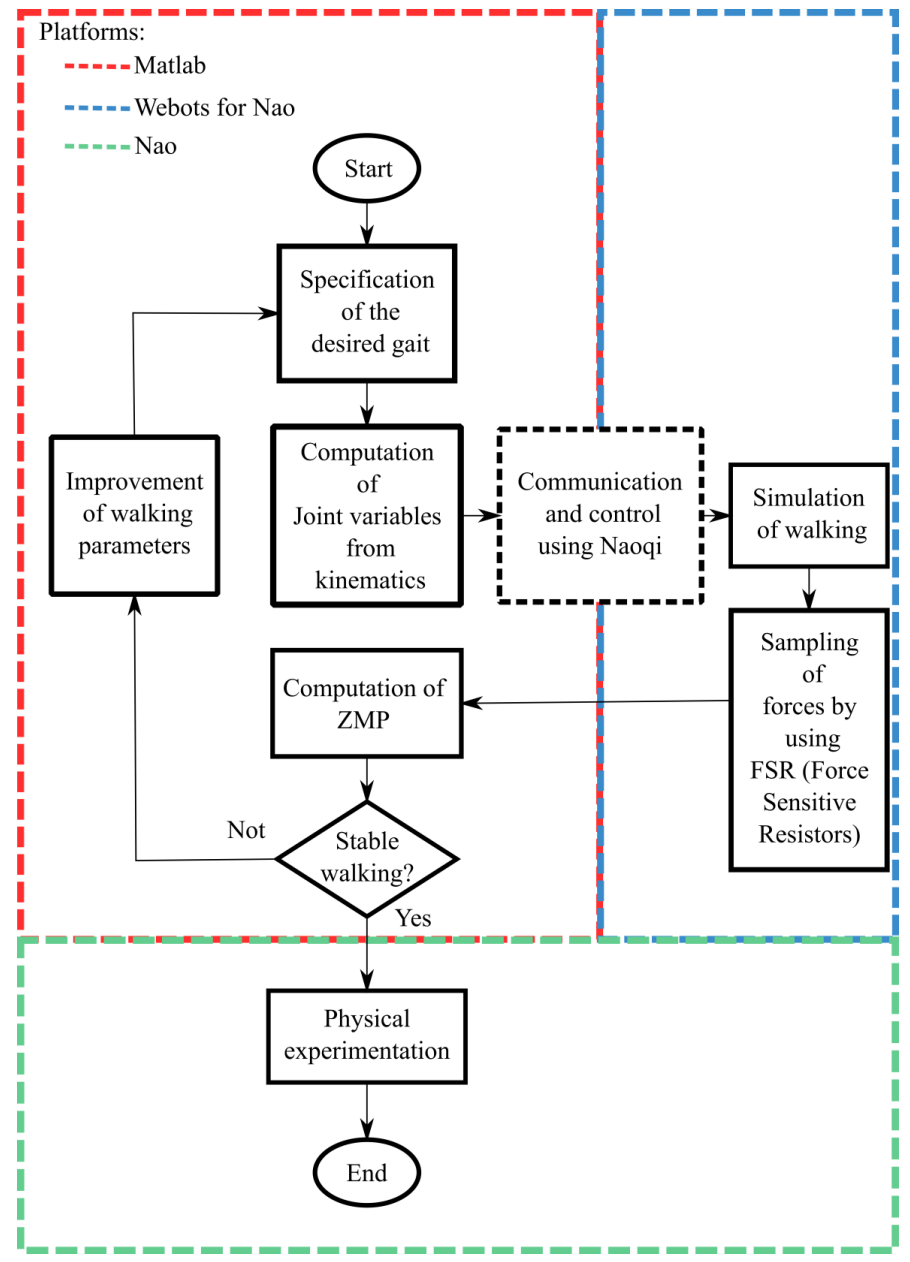

Fig. 4. Process applied to improve the stability of the Nao's walking

\section{Discussion of the results}

The numerical values obtained of parameters for the Simulated Aldebaran Gait (Case 1) are given in Table 1. It was perceived that the Aldebaran Gait completes double or triple oscillations during a step in motions of the pelvis and the oscillating foot for certain stages. That is why two or three values are identified for the parameters $\delta z_{p}, \delta y_{l f}$ and $\delta y_{r f}$ in Table 1. Such multiple oscillations during a step are not achieved in the cycloidal gait. On the other hand for the Aldebaran Gait it was observed that, before starting the walking, a preliminary motion was completed for the pelvis. Likewise, after stopping the motion of feet, an additional displacement of the pelvis is accomplished. Both issues occur in a situation of double support. Thus, in Table 1 for the Case 1 two supplementary segments of motion are considered: BW (before walking) and AS (after stopping) with periods of $1.35 \mathrm{sec}$. and $1.14 \mathrm{sec}$, respectively. The corresponding data are displayed in additional rows of Table $\mathbf{1 .}$

Simulations of Case 1 shown that not significative oscillations occur of the orientation coordinates of the Nao's torso. Such oscillations were of the order of $\pm 0.001^{\circ}$. So, it is considered that the motion of the torso is only translational. Furthermore, neither yaw nor roll rotations were observed in the oscillating foot. Only pitch rotations were appreciated on this body by an amount of about $\pm 19^{\circ}$. Besides, the results ratify that only single support phases arise in the Aldebaran gait. 
On the other hand, after the implementation of several tests in the process of Fig. 4 the sets of numerical values of the parameters for cycloidal gaits of cases 2 and 3 were established. Table 1 gives these values. By applying them the motions of the torso and the oscillating foot can be simulated. The results of such a simulation for Case 3 are observed in Fig. 5.

Several simulations were carried out to assess the stability of the robot in the three cases. For cases 1 and 3 the obtained samples of the ZMP on the footprint of the Nao are presented in Fig. 6. The corresponding values of the stability index $\delta_{k}^{*}$ are compared at each step in Fig. 7. The values of the stability index $\delta_{w}^{*}$ of the whole walking are compared for cases 1 and 3 in Fig. 8. Global improvements are perceived in the stability of the feet with the EDS Cycloidal Gait compared with the Simulated Aldebaran Gait. The values of the index $\delta_{w}^{*}$ go from $38.58 \mathrm{~mm}$ and $39.51 \mathrm{~mm}$ for $l f$ and $r f$, respectively, in Case 1, to 31.89 and $30.83 \mathrm{~mm}$ for $l f$ and $r f$, respectively, in Case 3 . These reductions signify improvements by $17.34 \%$ and $21.9 \%$ in the stability of the Nao with the left foot and the right foot, respectively.

Table 1

Numerical values $(\mathrm{mm})$ of parameters for cycloidal motions of cases study

\begin{tabular}{|c|c|c|c|c|c|c|c|c|c|c|}
\hline Case study & Smt* & $\delta x_{P}$ & $\delta y_{P}$ & $\delta z_{p}$ & $\delta x_{l f}$ & $\delta y_{l f}$ & $\delta z_{l f}$ & $\delta x_{r f}$ & $\delta y_{r f}$ & $\delta z_{r f}$ \\
\hline \multirow[t]{5}{*}{ 1. Simulated Aldebaran Gait } & $B W$ & -9.2 & 31 & $\begin{array}{c}0.7 \\
-3.5\end{array}$ & 0.0 & 0.0 & 0.0 & 0.0 & 0.0 & 0.0 \\
\hline & $S t$ & 13 & -51 & 1.7 & 0.0 & 0.0 & 0.0 & 38 & $\begin{array}{c}0.2 \\
-0.4 \\
0.1\end{array}$ & 20 \\
\hline & $\mathrm{Cr}$ & 37 & 41 & 1.2 & 77 & $\begin{array}{c}0.2 \\
-0.3 \\
0.1\end{array}$ & 20 & 77 & $\begin{array}{c}-0.1 \\
0.2 \\
-0.1\end{array}$ & 20 \\
\hline & $S p$ & 30 & 51 & $\begin{array}{c}-1.1 \\
0.8 \\
-0.3\end{array}$ & 0.0 & 0.0 & 0.0 & 38 & $\begin{array}{c}-0.1 \\
0.2 \\
-0.1\end{array}$ & 20 \\
\hline & $A S$ & 7.0 & -31 & 1.7 & 0.0 & 0.0 & 0.0 & 0.0 & 0.0 & 0.0 \\
\hline \multirow[t]{5}{*}{ 2. DS Cycloidal Gait } & $B W$ & 0.0 & 31 & 0.7 & 0.0 & 0.0 & 0.0 & 0.0 & 0.0 & 0.0 \\
\hline & $S t$ & 18 & -51 & -3.5 & 0.0 & 0.0 & 0.0 & 38 & 0.0 & 20 \\
\hline & $\mathrm{Cr}$ & 37 & 41 & 1.2 & 77 & 0.0 & 20 & 77 & 0.0 & 20 \\
\hline & $S p$ & 18 & 51 & -1.1 & 0.0 & 0.0 & 0.0 & 38 & 0.0 & 20 \\
\hline & $A S$ & 0.0 & -31 & 2.0 & 0.0 & 0.0 & 0.0 & 0.0 & 0.0 & 0.0 \\
\hline \multirow[t]{5}{*}{ 3. EDS Cycloidal Gait } & BW & 0.0 & 30 & 0.1 & 0.0 & 0.0 & 0.0 & 0.0 & 0.0 & 0.0 \\
\hline & $S t$ & 20 & -50 & -3.1 & 0.0 & 0.0 & 0.0 & 38 & 0.0 & 22 \\
\hline & $\mathrm{Cr}$ & 37 & 40 & 1.3 & 77 & 0.0 & 22 & 77 & 0.0 & 22 \\
\hline & $S p$ & 15 & 50 & -1.1 & 0.0 & 0.0 & 0.0 & 38 & 0.0 & 22 \\
\hline & $A S$ & 0.0 & -30 & 2.8 & 0.0 & 0.0 & 0.0 & 0.0 & 0.0 & 0.0 \\
\hline
\end{tabular}

Note: *-abbreviations: Smt-segment, BW-before walking, AS-after stopping, St-starting (SSP), Cr-Cruising, Sp - Stopping $(S S P)$

Finally, sequences of postures of the robot during the experimental walking are shown for cases 1 and 3, respectively, in Fig. 9 (Case 1) and Fig. 10 (Case 3). As observed in these figures, with respect to the path of reference the robot gets a better position at the end of the walking by employing the gait of Case 3 compared with the position gotten with the gait of Case 1. 

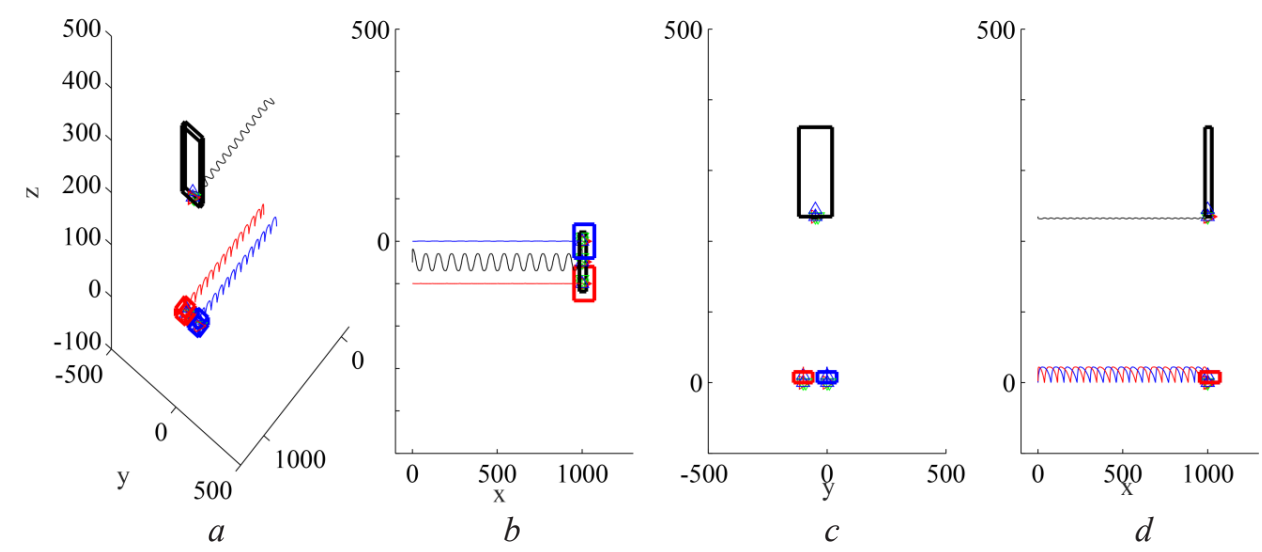

Fig. 5. Views of motion-simulation in Matlab $₫$ of the torso and oscillating feet for the EDS Cycloidal Gait (Case 3): $a$ - isometric; $b$-top; $c$ - front; $d$ - side

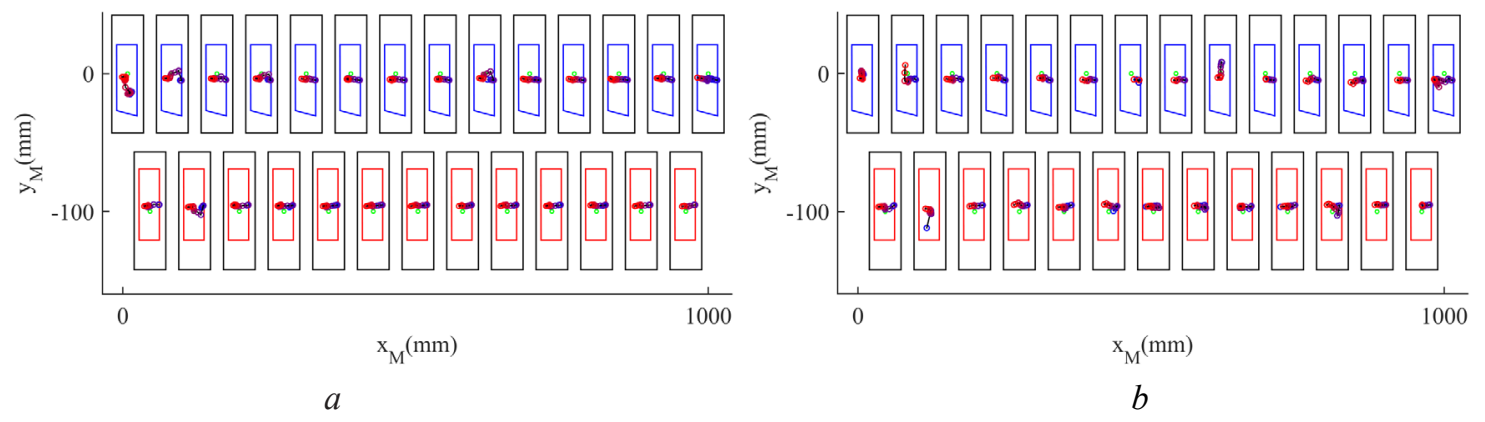

Fig. 6. Behaviour of ZMP on the footprints per step. Simulation in Matlab with data obtained from Webots for Nao: $a$ - Aldebaran Gait; $b$ - EDS Cycloidal Gait
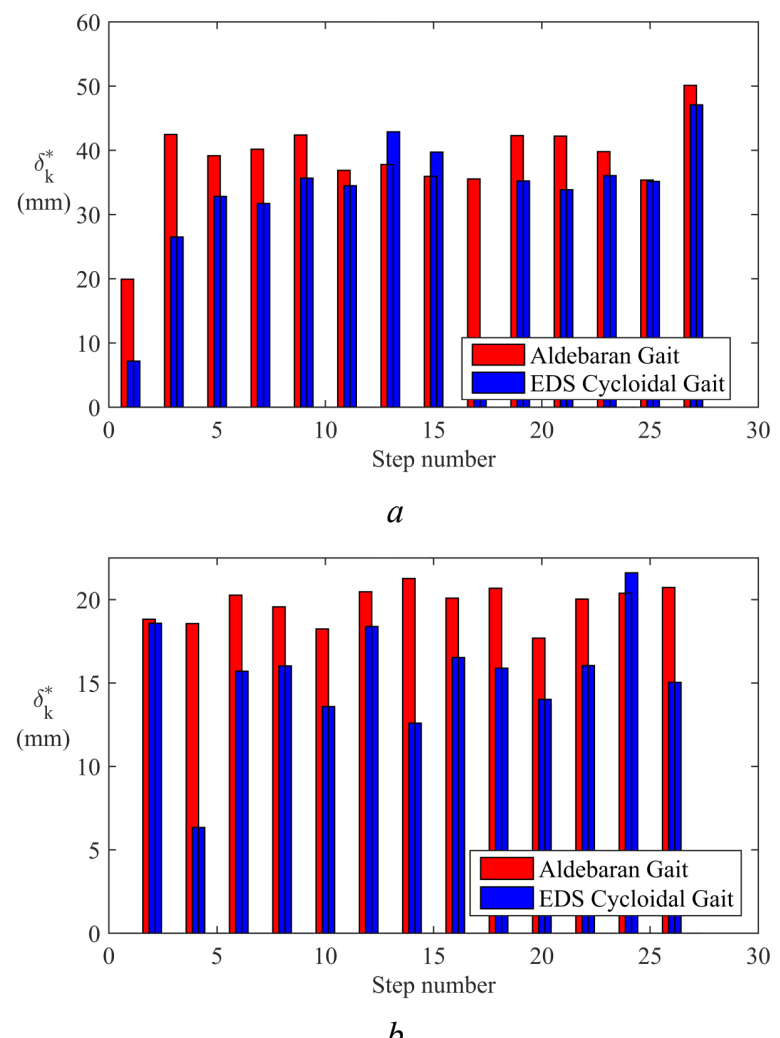

Fig. 7. Stability index $\delta_{k}^{*}$ at each step: $a$ - left foot; $b$ - right foot 


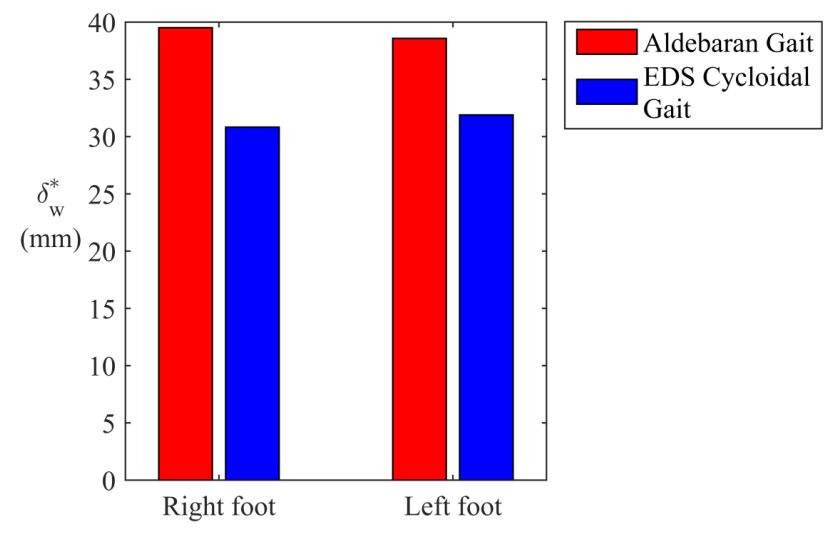

Fig. 8. Stability index $\delta_{w}^{*}$ for the whole walking

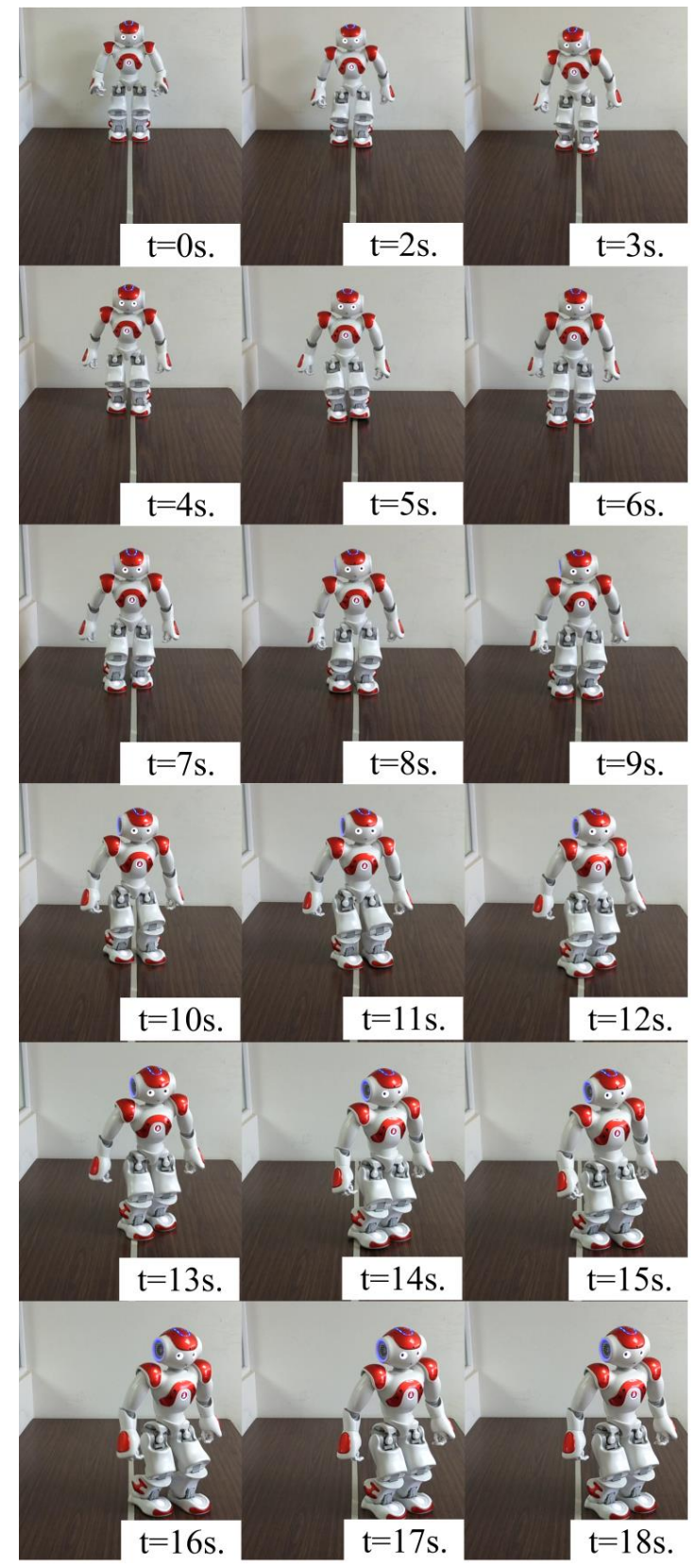

Fig. 9. Case 1. Postures of the Nao during the Aldebaran Gait. Front view. Only SSP are applied 


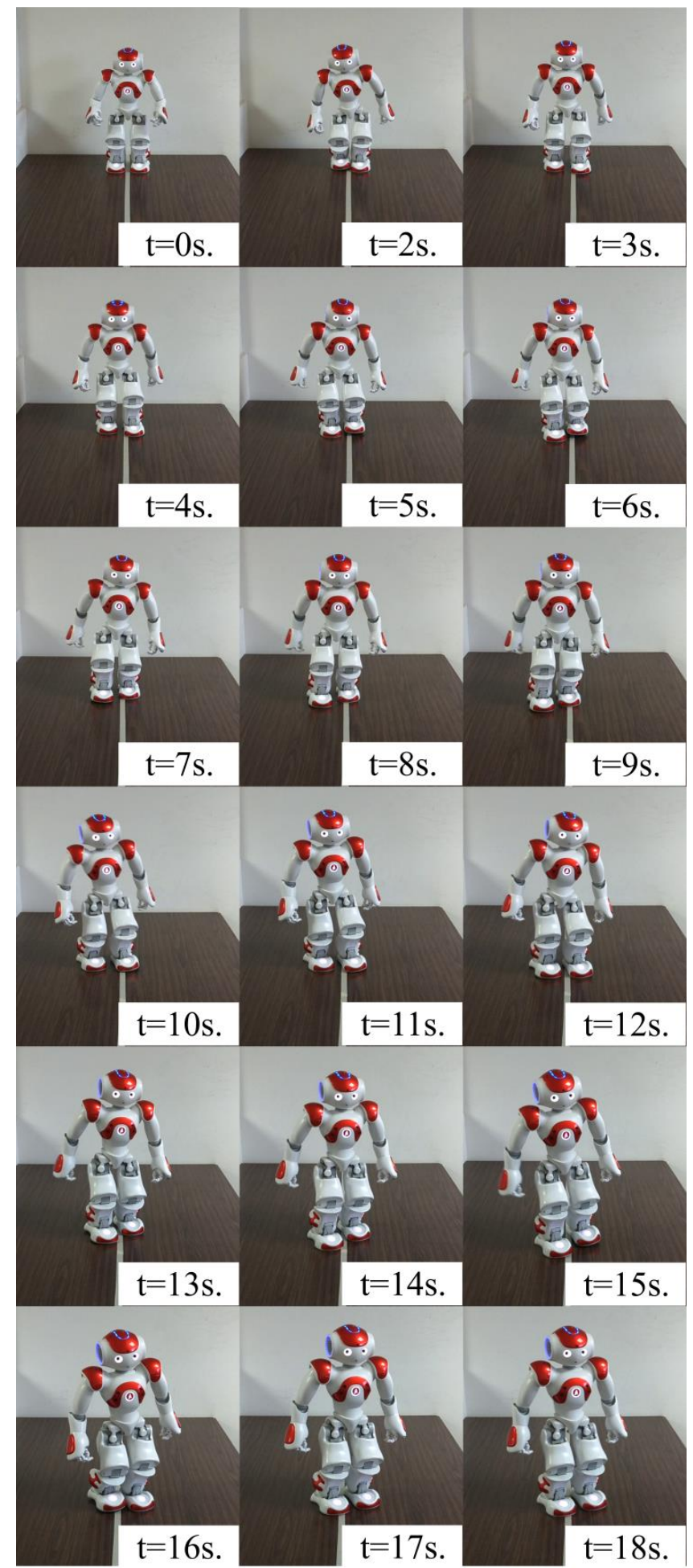

Fig. 10. Case 3. Postures of the Nao during the EDS Cycloidal Gait. SSP and DSP are employed

\section{Conclusions}

A strategy was proposed in this paper to improve the stability of the Nao humanoid robot during the walking. This robot has only 11 DOF in the legs. The proposed strategy is based on the integration of a double support phase and cycloidal motions to the gait of the robot. As a result, a significant improvement was gotten in the stability of the robot during the walking. The obtained simulations and the experimental results prove the enhancement. 
To measure the stability of the robot, the indices $\delta_{k}^{*}$ (for one step) and $\delta_{w}^{*}$ (for the whole walking) were introduced in the paper. These ones represent typical deviations of the ZMP from the centroid of the footprint of the robot. Consequently, the smaller are the indices the better is the stability of the robot during the walking. Substantial improvements were obtained in the index $\delta_{w}^{*}$ for the whole walking.

Thanks to the analysis accomplished in this work, it was appreciated that multiple transversal fluctuations occur in motions of both oscillating foot and the torso during the walking by employing the original Aldebaran Gait. Such fluctuations could be a source of instability and involve more energy consumption of the robot. That is why in future works studies of energy consumption related to the gait of the robot will be carried out. Furthermore, to improve the stability of the Nao's walking as much as possible by taking into account the approach introduced here, an optimization study will be also achieved in the future.

\section{Acknowledgments}

This work was supported by the Tecnologico Nacional de Mexico (TecNM) - Instituto Tecnologico de la Laguna, Ministry of Public Education (SEP) of Mexico, and the CONACyT (National Council of Research and Technology).

\section{References}

[1] Honda. Asimo the world's most advanced humanoid robot. Available at: http://asimo.honda.com/asimo-specs/

[2] Zhou, C., Li, Z., Wang, X., Tsagarakis, N., Caldwell, D. (2015). Stabilization of bipedal walking based on compliance control. Autonomous Robots, 40(6), 1041-1057. doi: https://doi.org/10.1007/s10514-015-9507-3

[3] Radford, N. A., Strawser, P., Hambuchen, K., Mehling, J. S., Verdeyen, W. K., Donnan, A. S. et. al. (2015). Valkyrie: NASA's First Bipedal Humanoid Robot. Journal of Field Robotics, 32 (3), 397-419. doi: https://doi.org/10.1002/rob.21560

[4] Kaneko, K., Kaminaga, H., Sakaguchi, T., Kajita, S., Morisawa, M., Kumagai, I., Kanehiro, F. (2019). Humanoid Robot HRP5P: An Electrically Actuated Humanoid Robot With High-Power and Wide-Range Joints. IEEE Robotics and Automation Letters, 4 (2), 1431-1438. doi: https://doi.org/10.1109/1ra.2019.2896465

[5] Tsagarakis, N. G., Caldwell, D. G., Negrello, F., Choi, W., Baccelliere, L., Loc, V. G. et. al. (2017). WALK-MAN: A High-Performance Humanoid Platform for Realistic Environments. Journal of Field Robotics, 34 (7), 1225-1259. doi: https://doi.org/ 10.1002/rob.21702

[6] Sugihara, T., Nakamura, Y. (2005). A Fast Online Gait Planning with Boundary Condition Relaxation for Humanoid Robots. Proceedings of the 2005 IEEE International Conference on Robotics and Automation. doi: https://oi.org/10.1109/robot. 2005.1570136

[7] De Magistris, G., Pajon, A., Miossec, S., Kheddar, A. (2017). Optimized humanoid walking with soft soles. Robotics and Autonomous Systems, 95, 52-63. doi: https://doi.org/10.1016/j.robot.2017.05.006

[8] Gouaillier, D., Hugel, V., Blazevic, P., Kilner, C., Monceaux, J., Lafourcade, P. et. al. (2008). The NAO humanoid: a combination of performance and affordability. arXiv e-Journal, Cornell University. Available at: https://arxiv.org/pdf/0807.3223v1.pdf

[9] Kajita, S., Hirukawa, H., Harada, K., Yokoi, K. (2014). Introduction to Humanoid Robotics. Springer Tracts in Advanced Robotics. doi: https://doi.org/10.1007/978-3-642-54536-8

[10] Fierro, J. E., Alfonso Pamanes, J., Moreno, H. A., Nunez, V. (2017). On the Constrained Walking of the NAO Humanoid Robot. Lecture Notes in Networks and Systems, 13-29. doi: https://doi.org/10.1007/978-3-319-54377-2_2

[11] Liu, J., Urbann, O. (2016). Bipedal walking with dynamic balance that involves three-dimensional upper body motion. Robotics and Autonomous Systems, 77, 39-54. doi: https://doi.org/10.1016/j.robot.2015.12.002

[12] Kajita, S., Kanehiro, F., Kaneko, K., Fujiwara, K., Harada, K., Yokoi, K., Hirukawa, H. (2003). Biped walking pattern generation by using preview control of zero-moment point. 2003 IEEE International Conference on Robotics and Automation (Cat. No.03CH37422). doi: https://doi.org/10.1109/robot.2003.1241826

[13] Pot, E., Monceaux, J., Gelin, R., Maisonnier, B. (2009). Choregraphe: a graphical tool for humanoid robot programming. ROMAN 2009 - The 18th IEEE International Symposium on Robot and Human Interactive Communication. doi: https://doi.org/ 10.1109/roman.2009.5326209 
[14] Webots: Open Source Robot Simulator. Available at: https://cyberbotics.com/

[15] Arias, L., Olvera, L., Pamanes, J. A., Nunez, J. V. (2014). 3D Walking cycloidal pattern for humanoids and its application to the Bioloid robot. Iberoamerican Journal of Mechanical Engineering, 18 (1), 03-22.

[16] Dombre, E., Khalil, W. (Eds.) (2007). Modeling, Performance Analysis and Control of Robot Manipulators. John Wiley \& Sons. doi: https://doi.org/10.1002/9780470612286

[17] Fierro, J. E., Pamanes, J. A., Arias, L. E. (2015). Walking of the humanoid robot Nao based on cycloidal motions. Proceedings of XXI International Annual Congress of the SOMIM, Mexican Society of Mechanical Engineering.

[18] Vukobratović, M., Borovac, B., Surla, D., Stokić, D. (1990). Biped Locomotion. Springer. doi: https://doi.org/10.1007/978-3642-83006-8

[19] Ren, L., Jones, R. K., Howard, D. (2007). Predictive modelling of human walking over a complete gait cycle. Journal of Biomechanics, 40 (7), 1567-1574. doi: https://doi.org/10.1016/j.jbiomech.2006.07.017

[20] Kajita, S., Kanehiro, F., Kaneko, K., Yokoi, K., Hirukawa, H. (2001). The 3D linear inverted pendulum mode: a simple modeling for a biped walking pattern generation. Proceedings 2001 IEEE/RSJ International Conference on Intelligent Robots and Systems. Expanding the Societal Role of Robotics in the the Next Millennium (Cat. No.01CH37180). doi: https://oi.org/ 10.1109/iros.2001.973365 